\title{
Correlative Microscopy Observation (3D EBSD + APT + TEM) on Intergranular Corrosion Behaviors in 316L Stainless Steel
}

Shao-Pu Tsai ${ }^{1 *}$, Peter Konijnenberg ${ }^{1,2}$, Stefan Zaefferer ${ }^{1}$, Nicolas Peter ${ }^{1}$, Baptiste Gault ${ }^{1}$, Kaori KawanoMiyata $^{3}$, Dayong $\mathrm{An}^{1}$ and Thomas Alister Griffiths ${ }^{1}$

1. Max-Planck-Institut für Eisenforschung, Düsseldorf, Germany.

2. Bruker Nano GmbH, Berlin, Germany.

3. Research and Development, Nippon Steel \& Sumitomo Metal Corporation (NSSMC), Japan.

* Corresponding author: s.tsai@mpie.de

AISI 316L is a corrosion-resistant stainless steel widely used in severe environments such as nuclear reactors. However, in real practice, AISI 316L suffers from significant intergranular stress corrosion cracking (IGSCC), which was reported to result from sensitization (Cr depletion at grain boundaries) [1, 2]. Researchers tried to employ grain boundary engineering to mitigate sensitization by thermomechanical treatments $[3,4]$. Therein, the main concept is to increase the proportion of special boundaries (i.e., coincidence site lattice (CSL) boundaries). $\Sigma 3$ grain boundaries are the most explored ones among all CSL boundaries, while others remain to be investigated in depth. An important shortcoming with this approach is that conventional 2D EBSD studies, which were mostly adopted in CSL analyses, revealed only three out of the overall five rotational degrees of freedom of grain boundaries.

Thus, in order to present a more complete picture of the corrosion - grain boundary character relationship, the current study aims at investigating the corrosion behaviors of specific grain boundaries in a sensitized 316L stainless steel by reconstructing 3D EBSD data sets (Fig. 1 (a)). Following the last slice of the set (Fig. 1(b)), interrupted corrosion tests (from 0 to 30 min with a time interval of 5 min) using a solution containing 10 vol. $\% \mathrm{HF}+90$ vol. $\% \mathrm{H}_{2} \mathrm{O}_{2}$ were then applied on the remaining material.

The corrosion results show that coherent $\Sigma 3$ boundaries and all $\Sigma 5$ boundaries never get corroded. Lowangle grain boundaries (with misorientation angle $<15^{\circ}$ ) show strong resistance to corrosion. In contrast, some CSL grain boundaries and random high angle grain boundaries (RHAGBs) were fractionally corroded depending on the applied corrosion time; CSL boundaries do not show a more pronounced corrosion resistance than RHAGBs. Of all the CSL boundaries investigated, all $\Sigma 7$ boundaries were significantly corroded (refer to Fig. 1(c-e)). Focused ion beam (FIB) machining was used to characterize the three-dimensional topography of corroded boundaries. It is found that corrosion only proceeds in the immediate neighborhood of the grain boundary. Thus, even though the corroded width is as narrow as $200 \mathrm{~nm}$, the corroded crack can continue as deep as $2 \mu \mathrm{m}$.

In order to address the distinct corrosion behaviors of $\Sigma 5$ and $\Sigma 7$ boundaries, both of which are lowindex CSL grain boundaries, atom probe tomography (APT) and scanning transmission electron microscopy (STEM) samples were target-prepared by FIB from grain boundaries with known plane indexing from the previous 3D EBSD observations. Earlier APT results from an investigation of 304L stainless steel [5] showed that the interstitial elemental segregation behavior of these boundaries was quite different, as shown in Fig. 2; the non-corroded $\Sigma 5$ GB showed a much lower concentration than its quicker-corroded $\Sigma 7$ counterpart. Detailed analyses by TEM show that the segregation can be further correlated with different atomic arrangements [6]. 
References:

[1] SM Bruemmer et al., Corrosion 48(1) (1992), p. 42.

[2] EA Trillo and LE Murr, Acta Mater. 47(1) (1999), p. 235.

[3] M Shimada et al., Acta Mater. 50(9) (2002), p. 2331.

[4] V Randle, Mater. Sci. Technol. 26 (2010), p. 253.

[5] D An et al., Acta Mater. 156 (2018), p. 297.

[6] We acknowledge financial support from NSSMC, Japan; Peter Konijnenberg is funded by Bruker Nano GmbH.
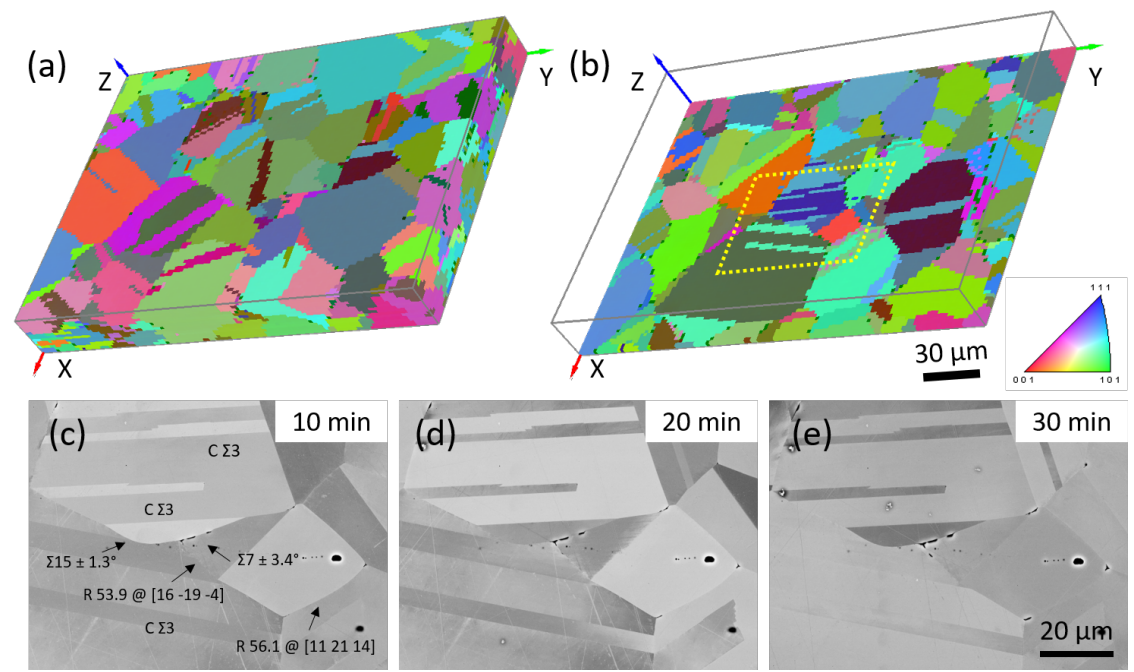

Figure 1. (a) Reconstructed 3D EBSD cube, where (b) is the last slice for interrupted corrosion tests (ce).
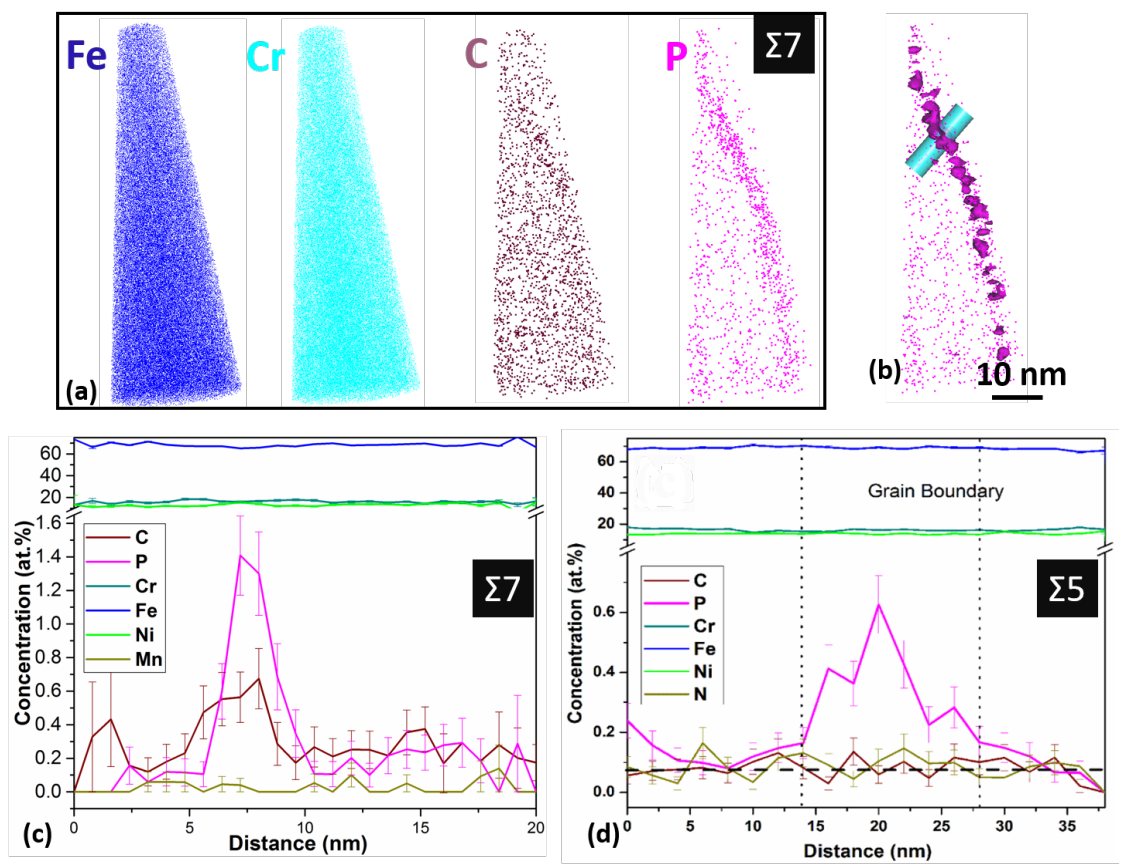

Figure 2. (a) 3-D APT reconstruction of atom positions showing enrichment of $\mathrm{P}$ and $\mathrm{C}$ atoms along $\Sigma 7$ grain boundary; (b) boundary region defined by using a P 0.4 at.\% iso-concentration surface from the same region in (a); (c) the concentration profiles for each atomic species obtained using a cylinder of 5 $\mathrm{nm}$ radius for $\Sigma 7 \mathrm{~GB}$ and (d) $\Sigma 5 \mathrm{~GB}$. 